\title{
Severe Neurological Signs Due to Hyponatremia in Patient with Acute Myeloid Leukemia; Ethiological Factors and Therapeutic Approach
}

Osman Yokus and Habip Gedik

Department of Infectious Diseases and Clinical Microbiology, Ministry of Health Bakırköy Sadi Konuk Training and Research Hospital, Istanbul, Turkey

*Corresponding author: Habip Gedik, Infectious Diseases and Clinical Microbiology Physician, Department of Infectious Diseases and Clinical Microbiology, Ministry of Health Bakırköy Sadi Konuk Training and Research Hospital, Istanbul, Turkey, Tel: 090-5053362770; E-mail: habipgedik@yahoo.com

Received date: Aug 11, 2015, Accepted date: Aug 18, 2015, Publication date: Aug 21, 2015

Copyright: ( 2015 Yokus Y, et al. This is an open-access article distributed under the terms of the Creative Commons Attribution License, which permits unrestricted use, distribution, and reproduction in any medium, provided the original author and source are credited.

\begin{abstract}
28-year-old male patient with acute promyelocytic leukemia developed pneumonia subsequent to chemotherapy. Geotrichum capitatum was isolated from sputum. Patient was admitted to intensive care unit due to respiratuar distress. Linezolid, imipenem, caspofungin and parenteral nutrition therapy (PNT) were initiated. Caspofungin was changed to voriconazole due to widespread reticulonodular and consolidated areas in chest computed tomography. During follow-up at intensive care unit, confusion, hyperkinesia, agitation and rigidity developed due to severe hyponatremia $(113 \mathrm{mEq} / \mathrm{L})$. Blood and urine osmolality with hyponatremia indicated inappropriate ADH syndrome. Linezolid and TPN were discontinued and isotonic solution was administered. Hyponatremia and clinical symptoms associated with penumonia recovered after 15 days of follow-up under voriconazole therapy that was administered for one month.
\end{abstract}

Keywords: Acute myeloid leukemia; Hyponatremia; Geotrichum capitatum; Inappropriate ADH syndrome

\section{Introduction}

Electrolyte imbalance is commonly seen in patients with acute leukemia. The most common cause of electrolyte abnormality is hypokalemia. But severe hyponatremia is infrequent. Metabolic studies defined disturbances as early increase of urinary sodium excretion, negative water clearance, and higher urine osmolality $[1,2]$. Central diabetes insipidus (CDI) develops in patients with acute myeloid leukemia (AML) because of infiltration of leukemic cells into the neurohypophysis or another reasons [3].

It is postulated that different etiologies cause this syndrome. In this case report, a patient who had acute myeloid leukemia (AML) and developed hyponatremia with all symptoms of inappropriate SIADH is being evaluated with probable causes and pathogenetic mechanisms of hyponatremia and CDI that is more likely to cause hyponatremia and related severe neurologic symptoms.

\section{Case}

28 year-old male patient was diagnosed with Acute Promyeloid Leukemia that includes translocation 15:17 that was detcted by FISH method and remission-induction chemotherapy (idarubicin and vesanoid) was initiated. Subsequent to second induction chemotherapy, profound neutropenia developed. Teicoplanin and imipenem were administered due to patient's complaints including fever $>38^{\circ} \mathrm{C}$, increased cough, phlegm, dyspnea. Chest computed tomography (CT) was revealed widespread reticulo-nodular increased density, ground-glass attenuation, and patch consolidations (Figure 1). Upper abdominal CT (Figure 2) revealed that multiple hypodense lesions in liver and spleen accordance with the fungal infection. Specimens including blood, urine, sputum, feces were cultured for microbiologic examination. Trimethoprim-sulfamethoxazole and caspofungin were added to treatment due to worsening clinical symptoms. Patients was followed up for two days at intensive care unit. Caspofungin was changed to voriconazole due to pneumonia caused by Aspergillus spp. Geotrichum capitatum was isolated from sputum and susceptible to voriconazole. Patient had recovered after ten days of treatment and control chest CT at first month of follow-up was normal (Figure 3). Laboratorial findings were $\mathrm{Na}$ with113 $\mathrm{mmol} / \mathrm{L}$, plasma osmolality with $235 \mathrm{mOsm} / \mathrm{kg} \mathrm{H}_{2} \mathrm{O}$ (normal range: 285-295), and urine osmolarity with $398 \mathrm{mOsm} / \mathrm{kg} \mathrm{H}_{2} \mathrm{O}$. Antidiuretic hormone $(\mathrm{ADH})$ could not be measured because of technical inconvenience. These results was consistent with inappropriate $\mathrm{ADH}$ syndrome and patient was consultated with nephrology. Isotonic solutions were administered. Serum Na decreased to $112 \mathrm{mmol} / \mathrm{L}$ and serum sale was added to isotonic solution $(500 \mathrm{cc})$ that was infused as adjusted to $25 \mathrm{cc} /$ hour. There was no definitive signs in brain MRI for diagnosis. Lumbar puncture to evaluate the cerbrospinal fluid could not be implemented due to incooperation with patient.

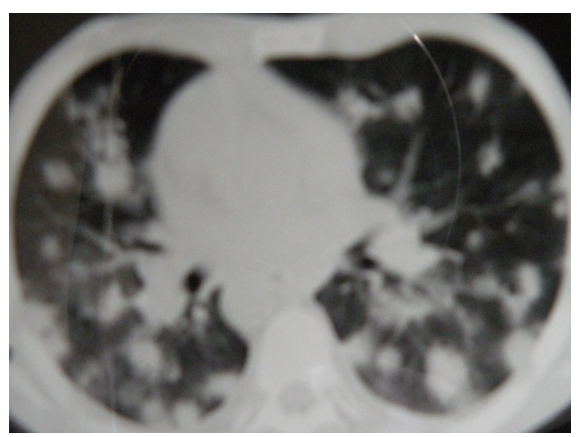

Figure 1: Chest CT scan (sagittal section) shows widespread reticulonodular and consolidated areas. 
Citation: Yokus O, Gedik H (2015) Severe Neurological Signs Due to Hyponatremia in Patient with Acute Myeloid Leukemia; Ethiological Factors and Therapeutic Approach. J Blood Disord Transfus S5: S5-001. doi:10.4172/2155-9864.1000S5-001

Page 2 of 3

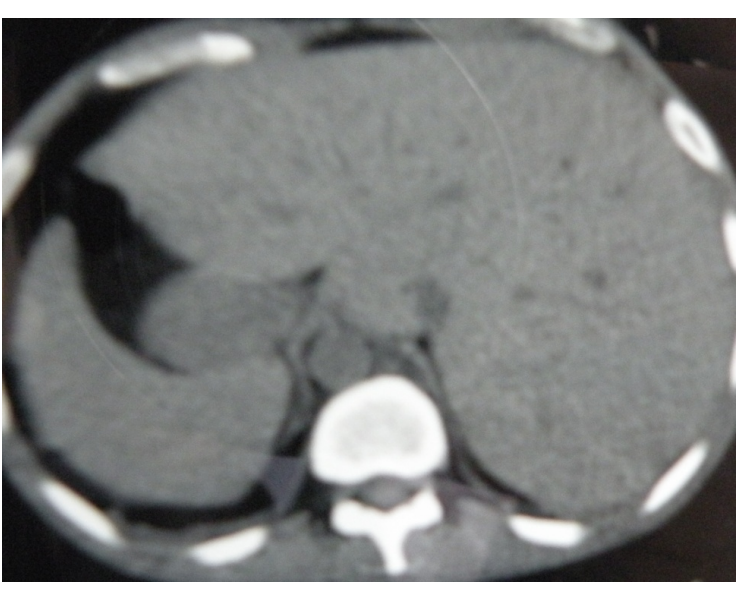

Figure 2: Upper abdominal CT reveals multiple hypodense lesions in the liver and spleen in accordance with the fungal infection.

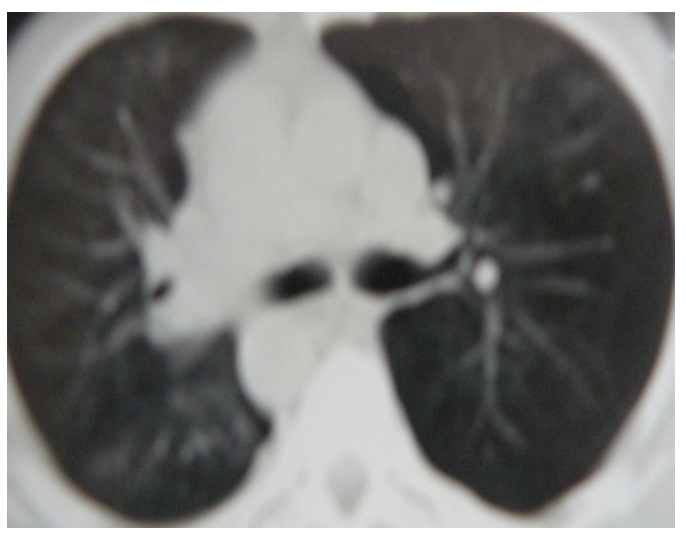

Figure 3: Control CT after one month of voriconazole treatment, resolution of lung infection was onitored.

Hyponatremia may occur due to inappropriate $\mathrm{ADH}$ syndrome and be a component of this syndrome due to the parenteral solutions, drugs, pulmonary infection or a combination of these factors. Parenteral nutrition and linezolid therapy were discontinued. Gliotic subcortical ischemic foci in the brain was captured by magnetic resonance imagination (Figure 4), central pontine myelosis was not reported. The patient was disoriented, agitated, anxious, depressed, fatigue, apathetic, confused in this period. There were also hyperkinesia, rigidity and confusion in examination. Sodium treatment was continued for about 10 days and $\mathrm{Na}$ level gradually increased to normal values $(135 \mathrm{mEq} / \mathrm{L})$. Patient gradually regained consciousness within 5 days. ROM (Range of motion), standing and strengthening exercises were worked out by the physiotherapy and rehabilitation physician. Voriconazole therapy was continued for 2 months. The patient was discharged with the request of patient and his parents. Exremity rigidity was observed minimal in controls, because of uncompleted physical therapy in this period and it had recovered after 2 months.

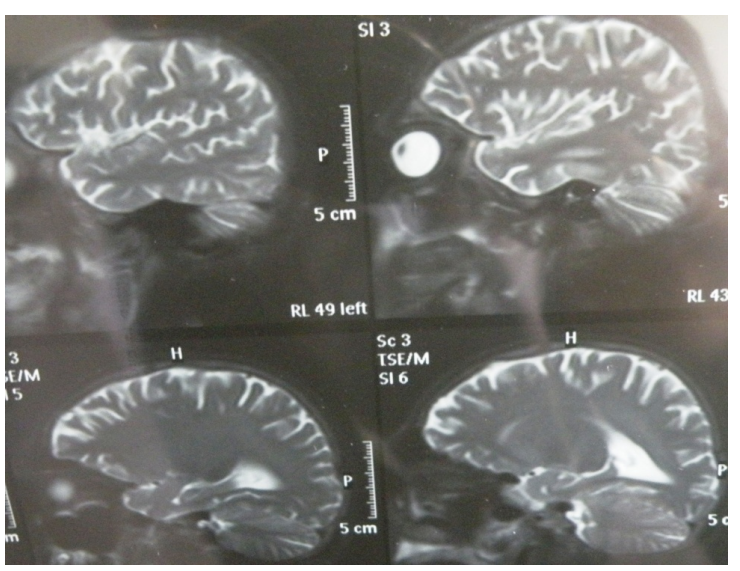

Figure 4: Cranial MRI shows Gliotic subcortical ischemic foci.

\section{Discussion}

Anorexia, nausea, vomiting, weakness, confusion, and coma generally develop in patients with hyponatremia associated with inappropriate $\mathrm{ADH}$ syndrome. Urine and blood osmolality should be measured for the diagnosis. Essential treatment is fluid restriction. In case whom acute neurological symptoms appear (Na level $110 \mathrm{mEq} / \mathrm{L})$, hypertonic solutions should be initiated. Furosemide and saline infusions are recommended as well. Due to hormones and cytokines secreted by the tumor, neuropathy and myopathy may occur. Hyponatremia can appear with signs and symptoms regarding central nervous system, such as hallucinations, disorientation and hypokinesia, dyskinesia in the extremities. Disatrophy developed in muscle as much as patient could not walk. Hyponatremia may occur due to malignancies, drugs, lung infections, etc. Hyponatremia may be a part of paraneoplastic syndrome in the course of malignancy with inappropriate $\mathrm{ADH}$ syndrome (SIADH). However, there are a few studies about hyponatremia and SIADH in patients with acute leukemia [4]. Although the reason of hyponatremia remains unclear in our case, pneumonia caused by Geotrichum capitatum, or medication (eg.linezolid) may be considered.

Geotrichum capitatum, a fungus causing systemic infections in immunosuppressed patients. G. capitatum infections most frequently occur $(80 \%)$ in patients with acute leukemia $[5,6]$. MIC is the lowest with a value of anti-fungal drug, voriconazole [7]. Therefore, this drug was initiated as soon as possible and the response was achieved in our patient.

Retrospective immunohistochemical analysis of blast cells was reported to be positive for antidiuretic hormone $(\mathrm{ADH})$ protein, which clearly demonstrated that the tumor cells produced ADH [8]. To measure serum $\mathrm{ADH}$ level is needed for accurate diagnosis. It could not be measured due to technical inconvenience. This diagnosis was supported with blood and urine osmolalities and patient was treated on the assumption of inappropriate $\mathrm{ADH}$ syndrome. Clinical symptoms and causes of hyponatremia in patient with acute leukemia are presented and discussed in line with literature.

Consequently, hyponatremia should be taken in to consideration in patients with hyperkinesia, rigidity and confusion under acute leukemia chemotherapy. 
Citation: Yokus O, Gedik H (2015) Severe Neurological Signs Due to Hyponatremia in Patient with Acute Myeloid Leukemia; Ethiological Factors and Therapeutic Approach. J Blood Disord Transfus S5: S5-001. doi:10.4172/2155-9864.1000S5-001

Page 3 of 3

\section{References}

1. Milionis HJ, Bourantas CL, Siamopoulos KC, Elisaf MS (1999) Acid-base and electrolyte abnormalities in patients with acute leukemia. Am J Hematol 62: 201-207.

2. Mir MA, Delamore IW (1974) Hyponatraemia syndrome in acute myeloid leukaemia. Br Med J 1: 52-55.

3. Sano S, Yamagami K, Morikawa T, Yoshioka K (2010) Myelodysplastic syndrome complicated by central diabetes insipidus and cerebral salt wasting syndrome with peculiar change in magnetic resonance images. Intern Med 49: 161-165.

4. Nakayama-Ichiyama S, Yokote T, Iwaki K, Takubo T, Tsuji M, et al. (2011) Syndrome of inappropriate antidiuretic hormone secretion associated with plasma cell myeloma. Br J Haematol 152: 125.

5. Buchta V, Otcenásek M (1988) Geotrichum candidum--an opportunistic agent of mycotic diseases. Mycoses 31: 363-370.
6. Girmenia C, Pagano L, Martino B, D'Antonio D, Fanci R, et al. (2005) Invasive infections caused by Trichosporon species and Geotrichum capitatum in patients with hematological malignancies: a retrospective multicenter study from Italy and review of the literature. J Clin Microbiol 43: 1818-1828.

7. Wildfeuer A, Seidl HP, Paule I, Haberreiter A (1998) In vitro evaluation of voriconazole against clinical isolates of yeasts, moulds and dermatophytes in comparison with itraconazole, ketoconazole, amphotericin B and griseofulvin. Mycoses 41: 309-319.

8. Nakayama S, Yokote T, Kobayashi K, Hirata Y, Akioka T, et al. (2009) Syndrome of inappropriate antidiuretic hormone secretion associated with acute myeloid leukemia with multilineage dysplasia. Endocrine 35: 290-292. 